\title{
Relating Vanishing Points to Catadioptric Camera Calibration
}

\author{
Wenting Duan*a, Hui Zhang ${ }^{\mathrm{b}}$, Nigel M. Allinson ${ }^{\mathrm{a}}$ \\ a Laboratory of Vision Engineering, University of Lincoln, Brayford Pool, Lincoln, U.K. LN6 7TS; \\ ${ }^{b}$ Dept. of Computer Science, United International College, 28, Jinfeng Road, Tangjiawan, Zhuhai, \\ Guangdong, China; Shenzhen Key Lab of Intelligent Media and Speech, PKU-HKUST Shenzhen \\ Hong Kong Institution, Shenzhen, China
}

\begin{abstract}
This paper presents the analysis and derivation of the geometric relation between vanishing points and camera parameters of central catadioptric camera systems. These vanishing points correspond to the three mutually orthogonal directions of 3D real world coordinate system (i.e. X, Y and Z axes). Compared to vanishing points (VPs) in the perspective projection, the advantages of VPs under central catadioptric projection are that there are normally two vanishing points for each set of parallel lines, since lines are projected to conics in the catadioptric image plane. Also, their vanishing points are usually located inside the image frame. We show that knowledge of the VPs corresponding to $\mathrm{XYZ}$ axes from a single image can lead to simple derivation of both intrinsic and extrinsic parameters of the central catadioptric system. This derived novel theory is demonstrated and tested on both synthetic and real data with respect to noise sensitivity.
\end{abstract}

Keywords: Vanishing points, catadioptric camera, calibration, projection geometry

\section{INTRODUCTION}

The extraction of information for camera parameters and scene geometry using vanishing points (VPs) are of importance to the application of 3D reconstruction, ego-motion, photogrammetry, etc. With the increasing trend of using catadioptric systems in various applications where a wide field of view is required, various techniques for calibration of catadioptric cameras are being developed. A catadioptric camera can be calibrated using two or more images of the same scene, e.g. Kang $^{1}$ and Micusik $^{2}$. Some researchers use planar grids. For example, Scaramuzza ${ }^{3}$ described the catadioptric image projection using a Taylor series expansion. The coefficients of the expansion model are obtained by solving a leastsquare linear minimisation problem. In $\mathrm{Mei}^{4}$ and Gasparini ${ }^{5}$ 's work, catadioptric homography is computed using images of planar grids. Factors such as misalignment of mirror and camera and camera-lens distortion can be taken into account. Another approach for calibrating catadioptric cameras is to use geometric properties such as line projections from a single catadioptric image. Ying ${ }^{6}$ demonstrated the use of line and sphere projections in central catadioptric camera calibration. Ying's work show that the projection of a sphere provides better conic fitting than line projection since sphere projection provide a larger portion of a conic. Hence a more accurate calibration resulted. However, the requirement of at least three sphere projections in some environments is not easy to satisfy. Previous research ${ }^{7,8}$ also shows that geometric properties of conics (line projections) in the catadioptric image enable camera calibration. These methods depend on the accurate fitting of at least three conics.

Vanishing points are the intersection of a set of parallel lines under camera projection. Since VPs are also a type of geometric feature, our work can also be categorised in the last approach (i.e. using geometric properties for camera calibration). The most similar work to ours in terms of using vanishing points for catadioptric camera calibration was done by Geyer and Daniilidis ${ }^{9}$. They proposed the use of two sets of parallel lines to locate the vanishing points and then developed the relation between the vanishing points and the camera intrinsic parameters. Nevertheless, this method is designed for para-catadioptric cameras. The para-catadioptric cameras are composed of paraboloid reflective and orthographic refractive. Under para-catadioptric projection, the parallel lines projected on the image plane are circular arcs with collinear centres when the aspect ratio is assumed to be unity. Compared to the hyper-catadioptric cameras (i.e. composed of hyperbolic reflective and perspective refractive), the mirror equation of the parabolic case is simpler and involve less parameters.

*wduan@lincoln.ac.uk; phone +44 (0)1522886955

Intelligent Robots and Computer Vision XXX: Algorithms and Techniques, edited by Juha Röning, David Casasent, Proc. of SPIE Vol. 8662, 86620C · (c) 2013 SPIE-IS\&T · CCC code: 0277-786/13/\$18 · doi: 10.1117/12.2008491 
- Parabolic mirror equation:

$$
\begin{gathered}
z=p-\frac{\left(x^{2}+y^{2}\right)}{4 p} \\
\frac{\left(\mathrm{z}-\frac{\mathrm{d}}{2}\right)^{2}}{\mathrm{a}^{2}}-\frac{\left(\mathrm{x}^{2}+\mathrm{y}^{2}\right)}{\mathrm{b}^{2}}=1
\end{gathered}
$$

The properties of VPs we derive in this paper are for the more complicated hyperbolic case. In fact, as described in Section 2, our derivation also covers the parabolic case since the parabolic case is one of the degenerate forms in central catadioptric cameras. In this paper, we analyse the image formation of VPs corresponding to the XYZ axes of the 3D real world coordinate system, and then demonstrate the calibration of central catadioptric systems through the detected VPs. In Section 2, the unifying model for catadioptric image formation and the projection of two parallel 3D lines are briefly explained. In Section 3, we analyse the geometric properties of vanishing points and how they relate to the camera parameters. In Section 4, we show how to use vanishing points for camera calibration. Section 5 outlines the experimental results for both synthetic and real data. Finally, we conclude in Section 6.

\section{UNIFYING MODEL}

The unifying model of central catadioptric projection developed by Barreto ${ }^{10}$ is shown in Figure 1. A 3D world point $\boldsymbol{X}$ is projected on the unit sphere at $\boldsymbol{X}_{C}$. The unit sphere is centre at the focal point of mirror and denoted as $\boldsymbol{O}$. The unifying model represents this transformation by a $3 \times 4$ matrix $\boldsymbol{P}$

$$
P=R[I \mid-C]
$$

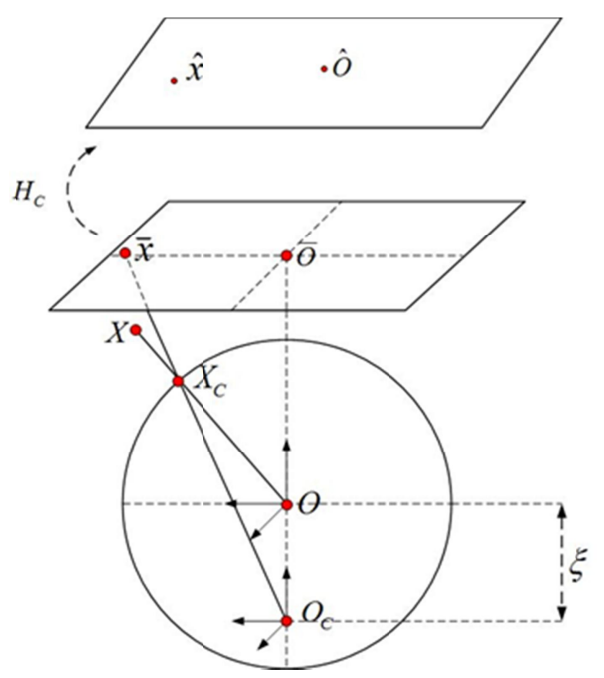

Figure 1. The unifying model for image formation of central catadioptric cameras.

After the transformation $\boldsymbol{X}_{C}=\boldsymbol{P} \boldsymbol{X}$, the point $\boldsymbol{X}_{C}$ is then mapped to the point $\overline{\boldsymbol{x}}$ in the sensor plane $\boldsymbol{\Pi}_{\infty}$. This transformation is modelled using function $\hbar$, in which the non-linearity of the mapping is embedded. The point $\boldsymbol{O}_{C}$ with coordinates $(0,0,-\xi)^{\mathrm{T}}$ is the other projection centre which re-projects the point $\boldsymbol{X}_{C}$ on the unit sphere to $\overline{\boldsymbol{x}}$ in the sensor plane $\boldsymbol{\Pi}_{\infty}$. The function $\hbar$ is written as

$$
\hbar(\boldsymbol{x})=\left[\begin{array}{c}
\frac{x}{\sqrt{x^{2}+y^{2}+z^{2}}} \\
\frac{y}{\sqrt{x^{2}+y^{2}+z^{2}}} \\
\frac{z}{\sqrt{x^{2}+y^{2}+z^{2}}}+\xi
\end{array}\right]
$$

Finally, a collineation $\boldsymbol{H}_{C}$ is applied to transform $\overline{\boldsymbol{x}}$ to obtain the point $\widehat{\boldsymbol{x}}$ in the catadioptric image plane, i.e. 
$\boldsymbol{H}_{C}$ is written as

$$
\widehat{\boldsymbol{x}}=\boldsymbol{H}_{\boldsymbol{C}} \overline{\boldsymbol{x}}
$$

$$
\boldsymbol{H}_{\boldsymbol{C}}=\boldsymbol{K}_{\boldsymbol{C}} \boldsymbol{R}_{\boldsymbol{C}} \boldsymbol{M}_{\boldsymbol{C}} \text { where } \boldsymbol{M}_{\boldsymbol{C}}=\left[\begin{array}{ccc}
\varphi & 0 & 0 \\
0 & -\varphi & 0 \\
0 & 0 & 1
\end{array}\right]
$$

$\boldsymbol{M}_{\boldsymbol{C}}$ changes according to the mirror type and shape, $\boldsymbol{K}_{\boldsymbol{C}}$ is the camera calibration matrix and $\boldsymbol{R}_{\boldsymbol{C}}$ is the rotation matrix of the mirror relative to the camera. The majority of the catadioptric sensors commercially available have their mirror accurately aligned with the camera, i.e. the conventional camera is not rotated with relation to the mirror surface. Therefore, the rotation matrix $\boldsymbol{R}_{\boldsymbol{C}}=\boldsymbol{I}$. Generally, the calibration of a central catadioptric system obtains the mirror parameters $\xi$ and the intrinsic parameter matrix $\boldsymbol{K}_{\boldsymbol{C}}$. The parameter $\varphi=\sqrt{1-\xi^{2}}$. For the parabolic mirror case, $\xi=1$ and the calibration is much easier. The lines under the projection of parabolic sensor are mapped to circles in the image plane if the aspect ratio of the camera is unity and the skew factor is zero ${ }^{9}$. Our target is the more complicated hyperbolic case where $\xi$ is unknown.

$$
\boldsymbol{K}_{\boldsymbol{C}}=\left[\begin{array}{ccc}
r . f_{e} & s & u_{0} \\
0 & f_{e} & v_{0} \\
0 & 0 & 1
\end{array}\right]
$$

$r$ is the aspect ratio, $f_{e}$ is the effective focal length, $s$ is the skew factor and $\left(u_{0}, v_{0}\right)$ is the principal point.

\section{CATADIOPTRIC PROJECTION OF VANISHING POINTS}

Section 2 described how a general point $\boldsymbol{X}$ in the 3D world is mapped to the image point $\widehat{\boldsymbol{x}}$ in the catadioptric image plane. In this section, the catadioptric projection of the three dominant vanishing points is introduced. The three ideal points $\boldsymbol{V}_{\boldsymbol{x}}, \boldsymbol{V}_{\boldsymbol{y}}$ and $\boldsymbol{V}_{\boldsymbol{z}}$ associated with the XYZ axes of the 3D world coordinate system are the so-called dominant vanishing points. They are firstly projected on the unit sphere by

$$
\boldsymbol{V}_{\boldsymbol{s}}=\boldsymbol{P}\left[\begin{array}{lll}
V_{x} & V_{y} & V_{z}
\end{array}\right]
$$

The centre of unit sphere $\boldsymbol{O}$ can be assumed to be located at $[0,0,0]$, then equation (3) becomes $\boldsymbol{P}=\boldsymbol{R}$. The ideal points $\boldsymbol{V}_{\boldsymbol{x}}, \boldsymbol{V}_{\boldsymbol{y}}$ and $\boldsymbol{V}_{\boldsymbol{z}}$ associated with the XYZ axes of the 3D world coordinate system are located at infinity along the direction of their associated axis. As shown in Figure 2, when $\boldsymbol{V}_{\boldsymbol{x}}, \boldsymbol{V}_{\boldsymbol{y}}$ and $\boldsymbol{V}_{\boldsymbol{z}}$ are projected to the unit sphere, their projections are simply $\boldsymbol{V}_{s x}=\left[\begin{array}{lll}1 & 0 & 0\end{array}\right], \boldsymbol{V}_{s y}=\left[\begin{array}{lll}0 & 1 & 0\end{array}\right]$ and $\boldsymbol{V}_{s z}=\left[\begin{array}{lll}0 & 0 & 1\end{array}\right]$, respectively.

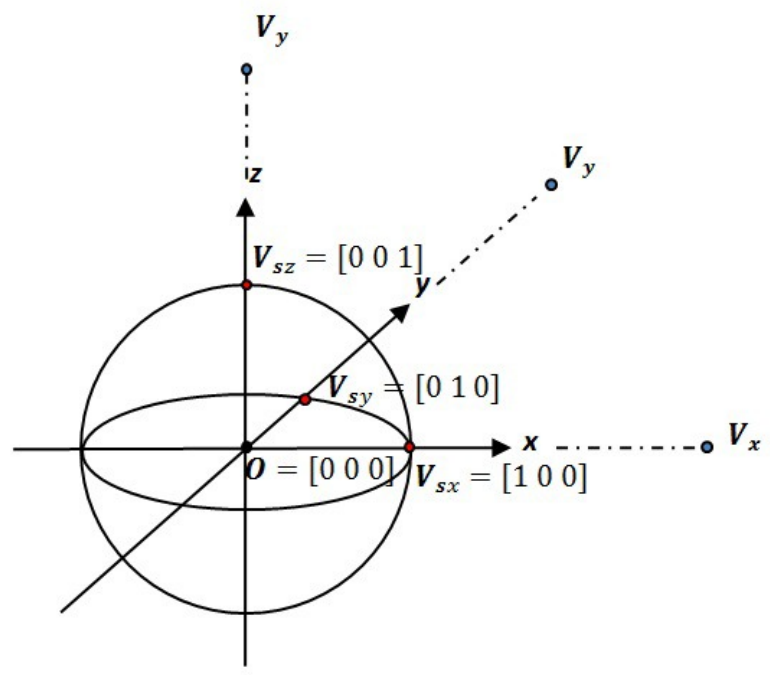

Figure 2. The projection of the three dominant VPs from 3D world to the unit sphere. 
We now have

$$
\boldsymbol{V}_{s}=\boldsymbol{R}\left[\begin{array}{lll}
1 & 0 & 0 \\
0 & 1 & 0 \\
0 & 0 & 1
\end{array}\right]=\left[\begin{array}{lll}
\boldsymbol{R}_{1} & \boldsymbol{R}_{2} & \boldsymbol{R}_{3}
\end{array}\right]
$$

where $\boldsymbol{R}_{1}, \boldsymbol{R}_{2}$ and $\boldsymbol{R}_{3}$ correspond to the columns of the rotation matrix $\boldsymbol{R}$. We then map the points on the sphere to the 2D canonical plane using equation (4). Since

$$
\boldsymbol{V}_{S}=\left[\begin{array}{lll}
R_{1 \_1} & R_{2 \_1} & R_{3 \_1} \\
R_{1 \_} 2 & R_{2 \_} & R_{3 \_} \\
R_{1 \_3} & R_{2 \_3} & R_{3 \_3}
\end{array}\right]
$$

and $\left\|\boldsymbol{R}_{1}\right\|=\left\|\boldsymbol{R}_{2}\right\|=\left\|\boldsymbol{R}_{3}\right\|=1$, the vanishing points $\boldsymbol{v}_{c}$ on the canonical plane equate to

$$
\boldsymbol{v}_{c}=\left[\begin{array}{ccc}
R_{1 \_1} & R_{2 \_1} & R_{3 \_1} \\
R_{1 \_2} & R_{2 \_} 2 & R_{3 \_2} \\
R_{1 \_3}+\xi & R_{2 \_3}+\xi & R_{3 \_3}+\xi
\end{array}\right]
$$

The last step in the image formation is to apply the collineation matrix $\boldsymbol{H}_{C}$ to $\boldsymbol{v}_{c}$. It is written as

$$
\boldsymbol{H}_{C}=\left[\begin{array}{ccc}
r \cdot f_{e} & s & u_{0} \\
0 & f_{e} & v_{0} \\
0 & 0 & 1
\end{array}\right]\left[\begin{array}{ccc}
\varphi & 0 & 0 \\
0 & -\varphi & 0 \\
0 & 0 & 1
\end{array}\right]
$$

If the dominant vanishing points in the image plane are denoted as $\boldsymbol{v}$, then in matrix form we have

$$
\boldsymbol{v}=\left[\begin{array}{ccc}
r . f_{e} & s & u_{0} \\
0 & f_{e} & v_{0} \\
0 & 0 & 1
\end{array}\right]\left[\begin{array}{ccc}
\varphi & 0 & 0 \\
0 & -\varphi & 0 \\
0 & 0 & 1
\end{array}\right]\left[\begin{array}{ccc}
R_{1 \_1} & R_{2 \_1} & R_{3 \_1} \\
R_{1 \_2} & R_{2 \_} & R_{3 \_} \\
R_{1 \_3}+\xi & R_{2 \_3}+\xi & R_{3 \_3}+\xi
\end{array}\right]
$$

Most commercially available cameras have square pixels with zero skew, hence we can assume $r=1$ and $s=0$. The vanishing points projected to the image plane can be expressed as

$$
\begin{aligned}
& v_{x}^{i+}=\frac{f_{e} R_{i \_1} \sqrt{1-\xi^{2}}}{R_{i_{3} 3}+\xi}+u_{0} \\
& v_{y}^{i+}=-\frac{f_{e} R_{i_{-} 2} \sqrt{1-\xi^{2}}}{R_{i_{-}}+\xi}+v_{0}
\end{aligned}
$$

where the index $i=1,2$ or 3 denote the VPs associated $\mathrm{X}, \mathrm{Y}$ and $\mathrm{Z}$ axes respectively. The superscript '+' indicate the vanishing points associated with the positive direction of $\mathrm{X}, \mathrm{Y}$ and $\mathrm{Z}$ axes.

As mentioned before, a set of parallel lines in the 3D world are projected to a set of conics intersecting at two vanishing points in the catadioptric image plane. We can use the same procedure to work out the other vanishing points which correspond to ideal points [- $\left.\begin{array}{llll}-1 & 0 & 0 & 0\end{array}\right],\left[\begin{array}{llll}0 & -1 & 0 & 0\end{array}\right]$ and $\left[\begin{array}{llll}0 & 0 & -1 & 0\end{array}\right]$ (i.e. ideal points lie at infinity towards the negative directions of $\mathrm{X}, \mathrm{Y}$ and $\mathrm{Z}$ axes).

\section{CAMERA CALIBRATION USING VANISHING POINTS}

Based on the relation between the VPs and camera parameters derived above, individual parameters can be obtained by following some simple equation manipulation. Figure 3 shows an example of catadioptric projection of lines. There are three groups of parallel lines with their associated $\mathrm{X}, \mathrm{Y}$ and $\mathrm{Z}$ axes directions. They are then mapped to the image plane 
as conics, each group of conics intersect at two vanishing points. For each pair of vanishing points detected in the image plane, a line $\boldsymbol{l}_{i}$ can be drawn to cross both points. If the vanishing points for each group of conics are expressed in homogenous coordinates $\boldsymbol{v}^{i+}=\left[\begin{array}{lll}v_{x}^{i+} & v_{y}^{i+} & 1\end{array}\right]^{T}$ and $\boldsymbol{v}^{i-}=\left[\begin{array}{lll}v_{x}^{i-} & v_{y}^{i-} & 1\end{array}\right]^{T}$, then $\boldsymbol{l}_{i}$ is obtained by the cross product of $\boldsymbol{v}^{i+}$ and $\boldsymbol{v}^{i-}$.

$$
l_{i}=v^{i+} \wedge v^{i-}
$$

The intersection of these three lines computed from each group of conics gives us the principal point $\widehat{\boldsymbol{O}}=\left[\begin{array}{lll}u_{0} & v_{0} & 1\end{array}\right]$. The principal point can be calculated using the cross product of any two lines obtained from equation (16).

$$
\widehat{\boldsymbol{O}}=\boldsymbol{l}_{1} \wedge \boldsymbol{l}_{2}
$$

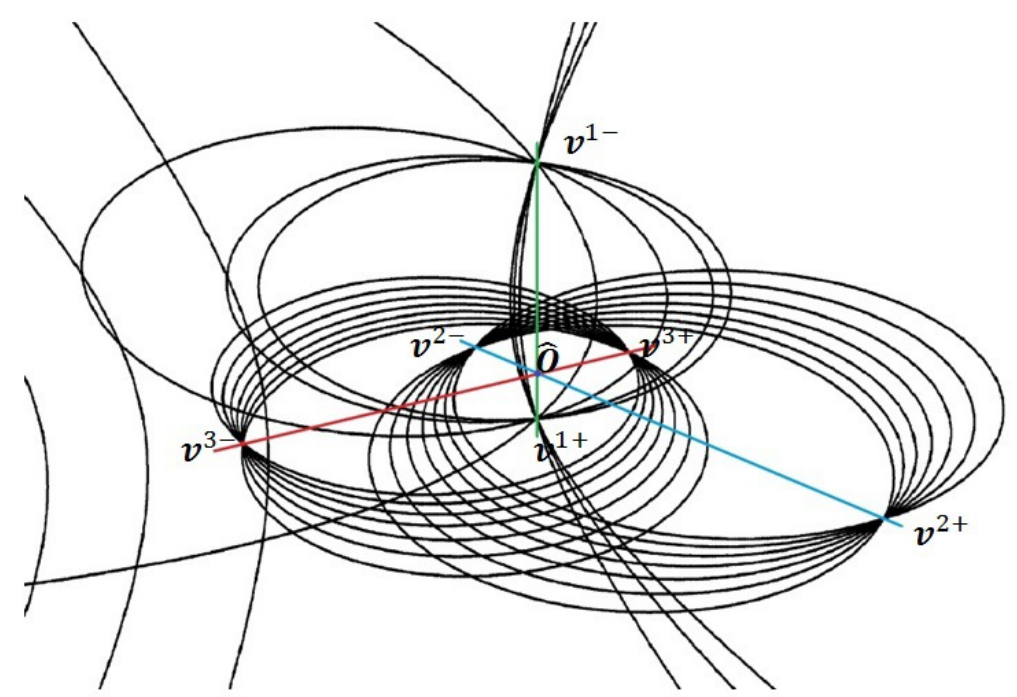

Figure 3. The projection of parallel lines in the image plane and their associated vanishing points.

Once the principal point is obtained, the columns of rotation matrix $\boldsymbol{R}$ can be derived. From equations (14) and (15), we have

$$
v_{x}^{i+}-u_{0}=\frac{f_{e} R_{i_{-} 1} \sqrt{1-\xi^{2}}}{R_{i_{-} 3}+\xi} \text { and } v_{y}^{i+}-v_{0}=-\frac{f_{e} R_{i_{-} 2} \sqrt{1-\xi^{2}}}{R_{i_{-}}+\xi}
$$

Divide the two equations in (18),

$$
\frac{v_{x}^{i+}-u_{0}}{v_{y}^{i+}-v_{0}}=\frac{v_{x}^{i-}-u_{0}}{v_{y}^{i-}-v_{0}}=-\frac{R_{i_{1} 1}}{R_{i_{2}}}
$$

If $a_{i}=\frac{v_{x}^{i+}-u_{0}}{v_{y}^{i+}-v_{0}}=\frac{v_{x}^{i-}-u_{0}}{v_{y}^{i-}-v_{0}}$ and we have three pairs of vanishing points, then there are

$$
a_{1}=-\frac{R_{1 \_1}}{R_{1 \_}}, \quad a_{2}=-\frac{R_{2 \_1}}{R_{2 \_}}, \quad a_{3}=-\frac{R_{3 \_1}}{R_{3 \_}}
$$

Here the properties involved with rotation matrix should be accounted for; that is the norm of each column or row of $\boldsymbol{R}$ is $1 ; \boldsymbol{R}$ is an orthogonal matrix. Now we have sufficient constraints to estimate the rotation matrix $\boldsymbol{R}$. Once $\boldsymbol{R}$ is obtained, $f_{e}$ and $\xi$ can be easily calculated. 


\section{EXPERIMENTAL RESULTS}

The calibration procedure illustrated in Section 4 is demonstrated and tested on both synthetic and real data with respect to noise.

\subsection{Synthetic data}

For this simulation, the calibration parameters of the catadioptric camera are set to: aspect ratio $\mathrm{r}=1$, skew factor $\mathrm{s}=0$, effective focal length $f_{e}=1000$, image centre $u_{0}=600$ and $v_{0}=400$, image size $1200 \times 800$ pixels and mirror parameter $\xi=0.92$. The rotation matrix is randomly generated using the formula

$$
\boldsymbol{R}=\left[\begin{array}{ccc}
\cos \theta \cos \delta & -\cos \phi \sin \delta+\sin \phi \sin \theta \cos \delta & \sin \phi \sin \delta+\cos \phi \sin \theta \cos \delta \\
\cos \theta \sin \delta & \cos \phi \cos \delta+\sin \phi \sin \theta \sin \delta & -\sin \phi \cos \delta+\cos \phi \sin \theta \sin \delta \\
-\sin \theta & \sin \phi \cos \theta & \cos \phi \cos \theta
\end{array}\right]
$$

Gaussian noise with zero mean and standard deviation varying from 0.0 to 5.0 pixels is added to each of the simulated vanishing points in the image plane. For each noise level, we perform 100 independent trials. The means and the standard deviations of the intrinsic parameters $f_{e}, \xi, u_{0}, v_{0}$ are computed and shown in Figure 4. The estimated principal points and mirror parameter are close to the ground truth as the noise level increase, therefore, it is reliable to use vanishing points to obtain the principal points and mirror parameters. Comparably, the focal length error gets larger when noise levels increase, this is due to its high magnitude (i.e. $10^{3}$ ) and the multiplication of uncertainty while calculating the focal length. However, the error in the focal length still stays reasonably small when the noise level is less than 4 pixels.

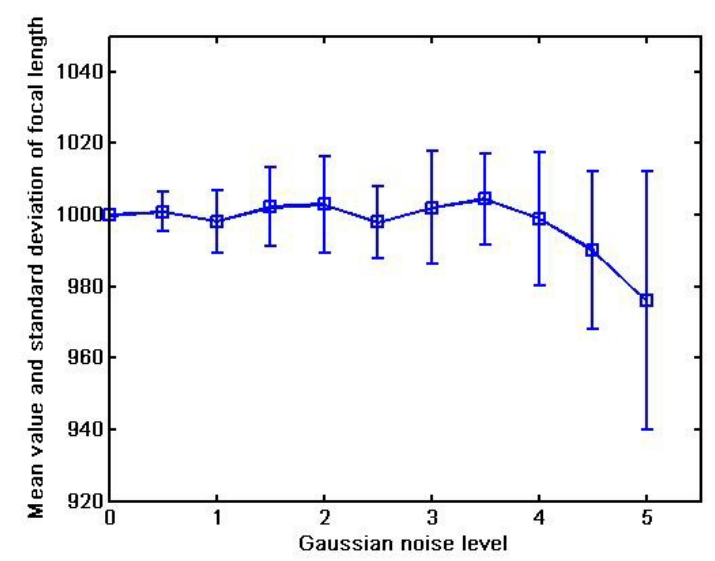

(a)

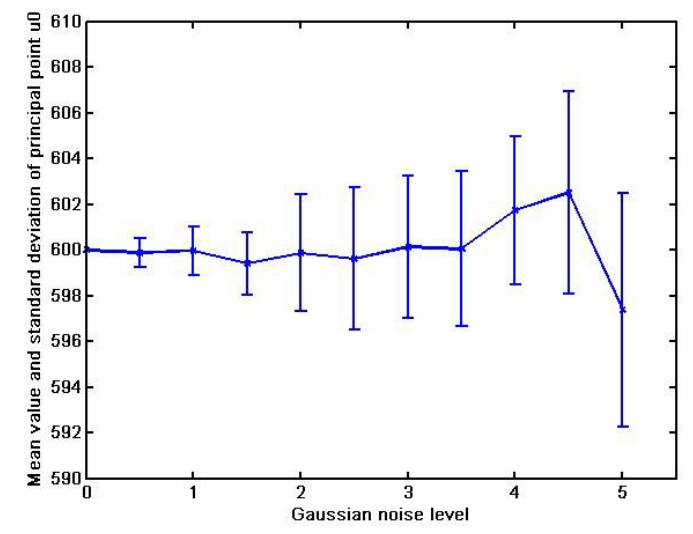

(c)

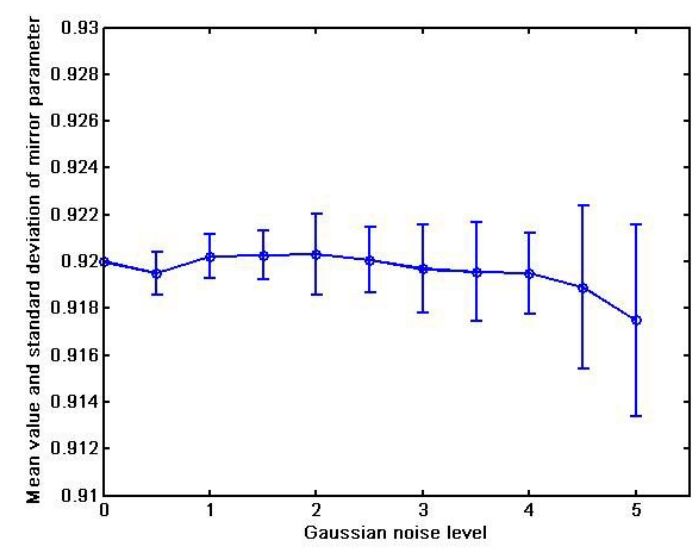

(b)

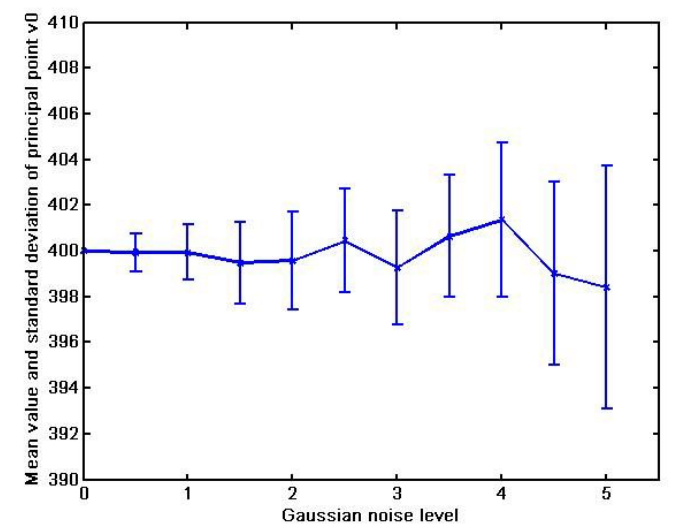

(d)

Figure 4. Experimental results of applying proposed calibration approach on synthetic data. 


\subsection{Real data}

The proposed method was also tested on real images. The conics are fitted to the extracted arc segments (i.e. the projection of straight lines) using the algorithm proposed by Sturm and Gargollo ${ }^{11}$; then vanishing points are detected as the intersection of 'parallel' conics. Figure 5 shows an example of conic fitting and vanishing points detection from a catadioptric image. We used a perspective camera with a hyperbolic mirror. The hyperbolic mirror employed is a commercially available unit called the 0-360 Panoramic Optic $\left(0-360^{\circ}\right.$.com, Carson City, USA), which has a vertical field of view (FOV) of $115^{\circ}$ and its vertical maximum visible angle above horizon is $52.5^{\circ}$. Images of five different manmade environments were taken. For each environment, images were repeated at six different locations. Each image was calibrated independently. Compared to the ground truth, the estimation results are as follows: the average error for the focal point is \pm 5.24 pixels, for the mirror parameter is \pm 0.021 , for the principal point $u_{0}$ is \pm 2.21 pixels, and $v_{0}$ is \pm 1.79 pixels. We know that only accurate calibration enables the rectification of the catadioptric image. In Figure 6 , some sample rectification results achieved using the calibrated parameters are shown.

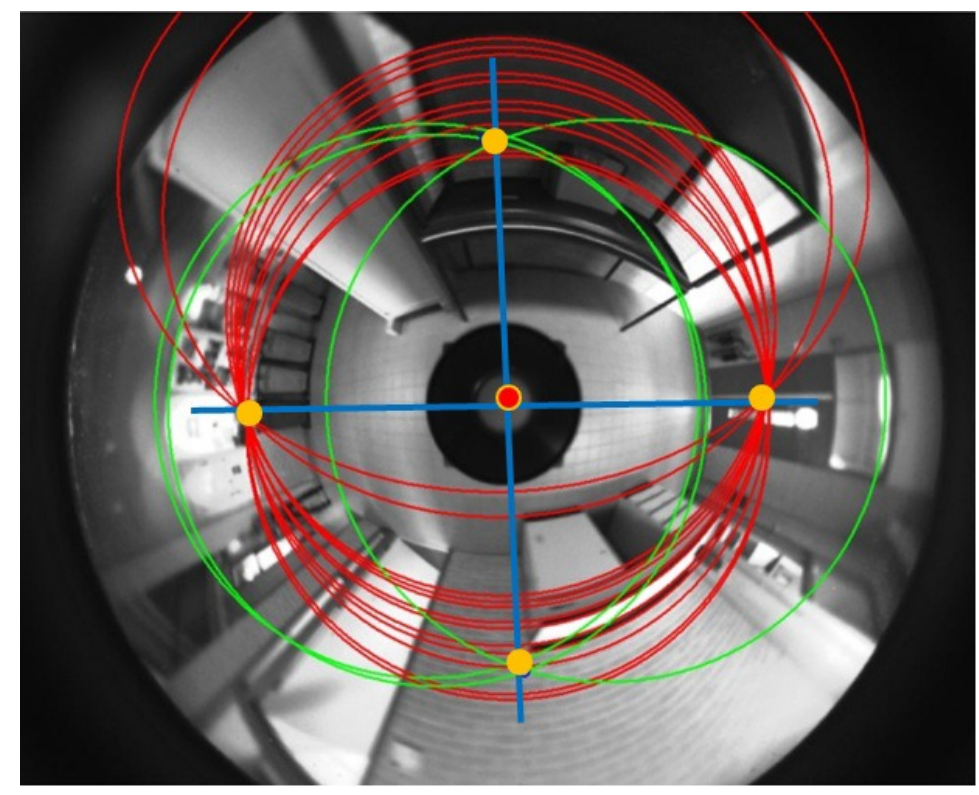

Figure 5, The detected vanishing points and principal points on real catadioptric image.
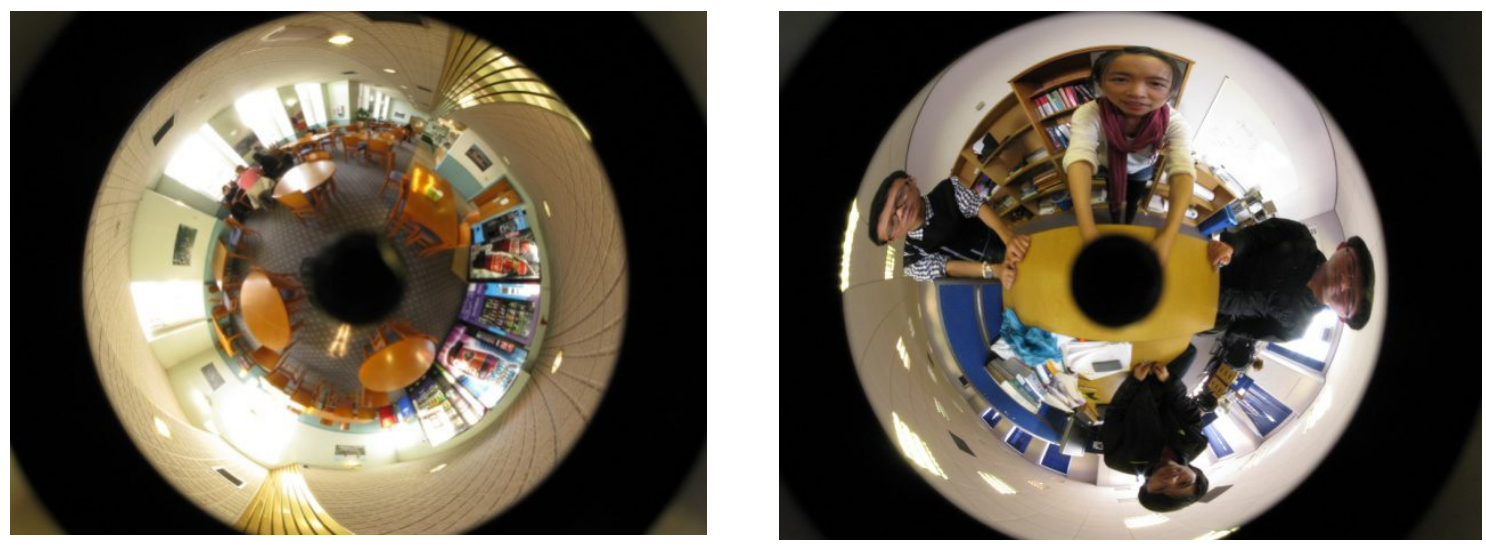

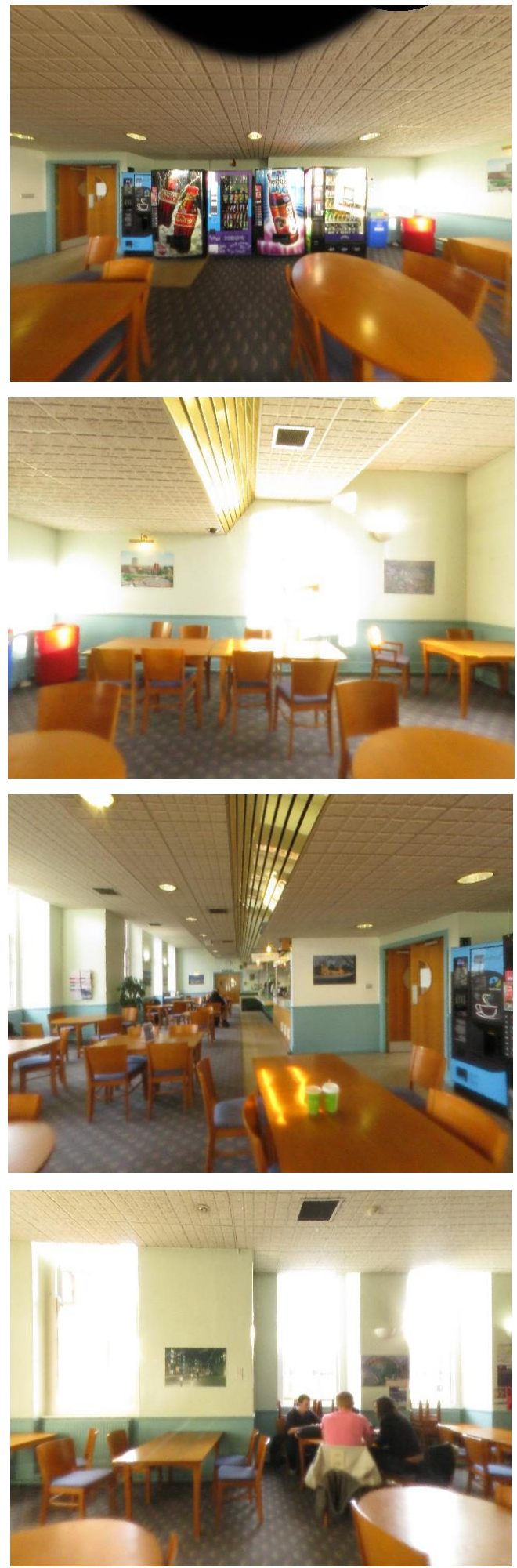
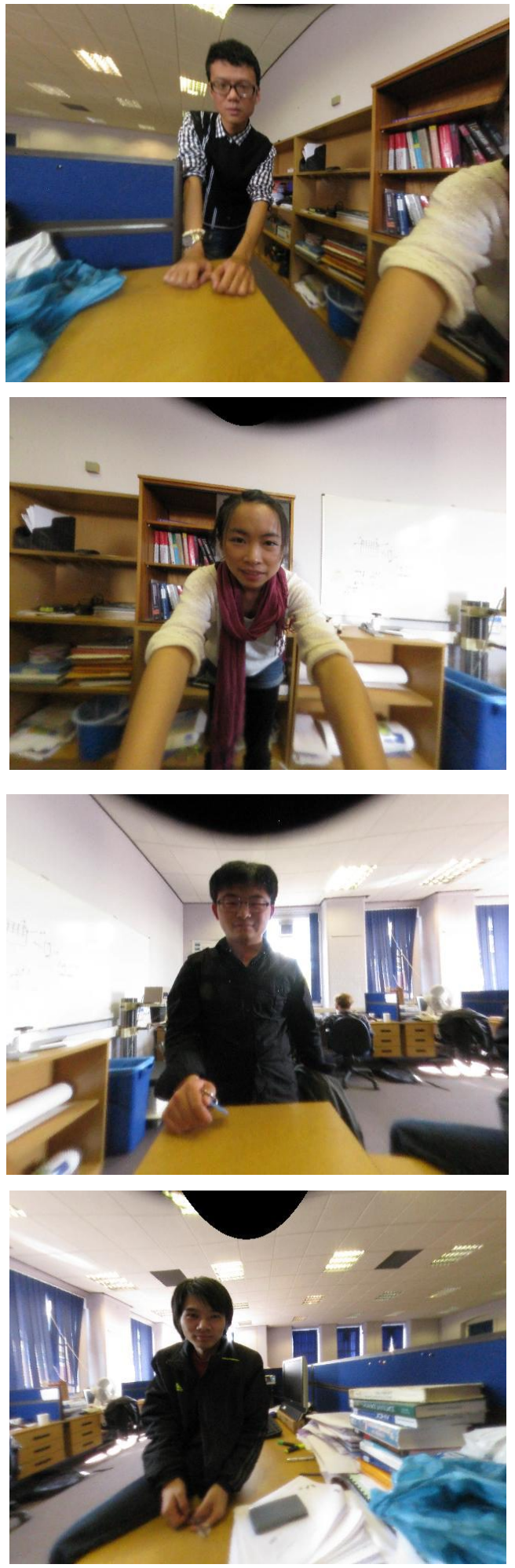

Figure 6. Visual assessment of calibration results: corrected perspectives of man-made environments and human faces. 


\section{CONCLUSIONS}

This paper first showed how vanishing points in the 3D world are projected onto the catadioptric image plane, and then we derived the relationship between vanishing points in catadioptric images and the camera parameters. Based on this derived relationship, we demonstrated that central catadioptric cameras can be calibrated using vanishing points which correspond to the three mutually orthogonal directions of the 3D real world coordinate system (i.e. $\mathrm{X}, \mathrm{Y}$ and $\mathrm{Z}$ axes). Compared to current calibration techniques ${ }^{12}$, the advantage of using vanishing points for calibration is that no planar grids are needed; no manual work is involved if automatic conic fitting and grouping are implemented; the calibration can be achieved from a single image. However, the disadvantage is that it can be less reliable than calibration using planar grids (if they are employed with care) since accuracy depends on the estimation of the detected vanishing points.

\section{ACKNOWLEDGEMENT}

The work is partially supported by the National Natural Science Foundation of China (Project No. 61005038) and United International College.

\section{REFERENCES}

[1] Kang, S.B., “Catadioptric self-calibration,” IEEE Conference on CVPR, 201-207 (2000).

[2] Micusik, B. and Pajdla, T., "Para-catadioptric camera auto-calibration from epipolar geometry," ACCV, (2004).

[3] Scaramuzza, D., Martinelli, A. and Siegwart, R., "A flexible technique for accurate omnidirectional camera calibration and structure from motion," IEEE Conference on Computer Vision Systems (2006).

[4] Mei, C. and Rives, P., "Single view point omnidirectional camera calibration from planar grids," IEEE Conference on Robotics and Automation, 3945-3950 (2007).

[5] Gasparini, S., Sturm, P. and Barreto, J.P., "Plane-based calibration of central catadioptric cameras," IEEE Conference on Computer Vision, 1195-1202 (2009).

[6] Ying, X. and Hu, Z., "Catadioptric camera calibration using geometric invariants," IEEE Transactions on Pattern Analysis and Machine Intelligence, Vol.26, 1260-1271 (2004).

[7] Barreto, J. and Araujo, H., "Direct least square fitting of paracatadioptric line images," Computer Vision and Pattern Recognition Workshop, 78-87 (2003).

[8] Ying, X. and Zha, H., "Simultaneously calibrating catadioptric camera and detecting line features using hough transform," IEEE Conference on Intelligent Robots and Systems, 1343-1348 (2005).

[9] Geyer, C. and Daniilidis, K., "Catadioptric camera calibration," IEEE Conference on Computer Vision, 398-404 (1999).

[10] Barreto, J., "General central projection systems, modelling, calibration and visual servoing," PhD thesis, Dept. of Electrical and Computer Engineering, University of Coimbra (2003).

[11] Sturm, P. and Gargallo, P., "Conic fitting using geometric distance," ACCV, 373-383 (2007).

[12] Puig, L., Bermudez, J., Sturm, P. and Guerreto, J., "Calibration of omnidirectional cameras in practice: A comparison of methods," Computer Vision and Image Understanding 116, 120-137 (2012). 